\title{
Research on the Music Culture Form of the Intangible Cultural Heritage in Manai Guozhuang
}

\author{
Yiran Feng \\ Southwest Minzu University, Chengdu, China \\ Email: 10236849@qq.com
}

How to cite this paper: Feng, Y. R. (2021). Research on the Music Culture Form of the Intangible Cultural Heritage in Manai Guozhuang. Open Journal of Social Sciences, 9, 42-52.

https://doi.org/10.4236/jss.2021.912003

Received: November 4, 2021

Accepted: December 4, 2021

Published: December 7, 2021

Copyright $\odot 2021$ by author(s) and Scientific Research Publishing Inc. This work is licensed under the Creative Commons Attribution International License (CC BY 4.0).

http://creativecommons.org/licenses/by/4.0/

\section{(c) (i) Open Access}

\begin{abstract}
Manai Guozhuang has a history of one thousand years, which was included in the protection list of "national intangible cultural Heritage" in December 2008. The songs originally had more than 200 copies, which were widely used by Tibetans in Jiarong for building houses, weddings, funerals, planting and harvest. They were banned for 10 years during the "Breaking the Four Old Traditions" movement of the Cultural Revolution. After being gradually restored, more than 100 copies remained and have been handed down to this day. This paper analyzes and studies the historical origin and musical structure of Manai Guozhuang (including lyrics, tonality, pitch, range and material structure), so as to explore the cultural ideology of the local Jiarong Tibetan (such as the reflection of the Buddhist concept of reincarnation and the "three kingdoms" concept of Bon religion in the musical structure), and explore the value and charm of Manai Guozhuang songs.
\end{abstract}

\section{Keywords}

Intangible Cultural Heritage, Manai Guozhuang, Music Form

\section{Introduction}

Manai township is located in the south of Jinchuan County, with an average elevation of 2180 meters and an annual average temperature of about $15^{\circ} \mathrm{C}$. It is 57 kilometers away from Jinchuan county and 35 kilometers away from Danba county. In December 2008, Manai Guozhuang was included in the "National Intangible Cultural Heritage" protection list. Local people have focused on the inheritance and protection with specialized professors. In July 2013, the author was lucky enough to find 7 inheritors, among whom the youngest heir Qian Fu- 
quan was 48 years old and the oldest Zhong Fuquan was 70 years old. The author learned that Manai Guozhuang music had more than 200 copies. Now, there are a few people over 80 years old can sing the remaining 100 copies. So far, there are few research results, as of November 2021, there are altogether 7 papers with the keyword "Manai Guozhuang" in their titles in THE CNKI database. The representative "Analysis of Manai Guozhuang" and "An Overview of Jiarong Tibetan Folk Dance-The Cultural Connotation of Manai Guozhuang" mainly focus on the general situation, dance formula, clothing and cultural connotation and social function research. The Manet Guozhuang Field Survey explores the inner relationship between the art form and culture and folk customs by using the method of artistic anthropology, and brief lists the collection of 15 copies. It can be seen that the academic research on Manet's guozhuang music is still in a relatively weak state.

\section{The Origin of Manai Guozhuang}

1) Cultural origin

Before the introduction of Tibetan Buddhism, the local people believed in The Bon religion, which regarded that the world was composed of three realms, namely, heaven, earth and underground. Then people were in the middle of the three realms. Therefore, there was a teaching ritual of "worshiping the god of heaven on the upper side, resuming people's houses in the middle sed, shaking ghosts and gods in the lower side" (Ga, 2005). 7 After the introduction of Tibetan Buddhism, The mutual absorption and compatibility of Buddhism and Bon religion made the two become "inseparable". Manai Guotzhuang exists in such a context of Bon Buddhism fusion. In the music structure, there is not only the consciousness of "three realms" in Bon, but also the causal theory of reincarnation.

According to legend, Manai area was once the site of ancient Eastern Female Kingdom. "The Old Tang Book" records that the country "respects women and despises men, women wear expensive clothes with male guards." (Mao, 2011) Although the ancient Eastern Female Kingdom has disappeared, the cultural heritage has been absorbed. In Manai Guozhuang, the cultural heritage of female supremacy and male inferiority can still be found.

2) Three legends of origin

a) It is said that Manai Guozhuang originated from the celebration held when the folk hero Amigudong (King Gesar) succeeded in conquering the devil, and later gradually expanded to the celebration or ceremony of welcoming the upper figures (Yang, 2009); b) Manai Guozhuang originated from the period of ancient Eastern Female Kingdom. It is a form of artistic performance to please the queen with strong matriarchal heritage. c) In order to celebrate the completion of the largest Infested Zhonglading Bon temple in eastern Tibet, which was renamed as The Gelug School Temple-Guangfa Temple after Qianlong in the Qing Dynasty, monks and people carried on the celebration in a form of song and dance. 
Infested Zhonglading Temple is located beside Dadu River in Anning town adjacent to Manai. There is a folk saying that Jiarong Guozhuang dance originated from Manai Guozhuang, and every village has teachers engaged in communication and teaching, which broadcasts Manai Guozhuang along Dadu River to Ganzi, Qinghai and Jiuzhai.

\section{Manai Guozhuang Music Structure}

The lyrics in Manai Guozhuang are said to be the ancient Xiangxiong language, and some people call it the local "Situ Dialect" (a dialect in Tibetan), which is quite different from the Tibetan language of Jiarong. According to the author's investigation, the local inheritors of the Situ Dialect could not translate the lyrics sentence by sentence, only knowing the general idea of the lyrics and the meaning of individual words. Therefore, the lyrics should be the ancient Xiangxiong language, whose text and the example are marked by romanization.

Manai guozhuang is divided into big Guozhuang (Dalgardi) and small Guozhuang (Dalgaryin). Big Guozhuang is more serious and solemn, while small Guozhuang is usually performed by young people, which is more free and cheerful, including love, entertainment and other relatively light and cheerful themes. In almost all the starts, the man sings first and the woman sings along. From the musical form to distinguish the size of Guozhuang, it depends on the melody at the end of the line:

Transcription 1: Small Guozhuang (Dalgaryin)

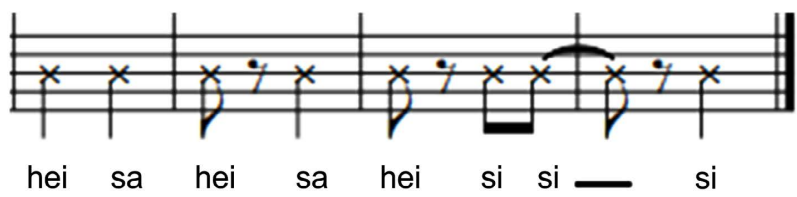

If there has such pattern, it is a small Guozhuang, if there is no such pattern, it is big Guozhuang. Big Guozhuang is relatively solemn and serious, while half squat kneeling in the end, which fully reflects the ritual and etiquette.

The whole procession is usually led by the oldest person (the venerable dancing leader), there are also more special circumstances, for example, marriage Guozhuang "Ni Mo Na Qi" (mother's milk), is led by the bride's uncle; "Nial ce Pangdang" is performed by the master stonemason who repaired the house. Then there is the equally important second command, which is taken by the best Guozhuang dancer in the team. Before the start, a grand opening ceremony should be held. Then the respected elders will inform the gods of the upcoming Guozhuang. Every year in the eighth month of the lunar calendar when harvesting 15 kinds of wheat, more than 30 families to hold the dinners in turn, jumping Guozhuang all night; In commemoration of the third anniversary of the death for their loved ones, they will jump for three days and three nights, meaning to inform the deceased that their loved ones are doing well and can safely enter the next cycle. Guozhuang runs through the life of The Jiarong Tibetan in 
Manai, they will jump when repairing house, celebrating happy events and farming.

A large circle dance team does not need to be organized, but relies on a long song to gather partners, and as soon as someone sings slowly and clearly, the Jiarong Tibetans of Manai will join in the procession. In the original Guozhuang, the long-drawled sound has the function of calling partners. But as Manai Guozhuang was listed as an intangible cultural heritage, it began to be known by more people, the public has incomprehension on the long and slow original ecological music, so that the Manai Guozhuang, which is used for performance purposes, is usually an "improved version", shortening the duration of the original long notes and standardizes the original free beat into a normative one that is more acceptable under the aesthetic standards.

In Manai guotang, when a female leading dancer presents a hada or a handkerchief to a male leading dancer, the male must crouch at his feet, palms up, and respectfully take the hada or a handkerchief, making three obeisance. If compare leader to a "command", then handkerchief plays the role of the "baton", during the process of Guozhuang, the leader would sometimes lift the handkerchief above his head and shake it clockwise or counterclockwise, which becomes an "indication" for the dance team to change the formation in the direction. At the beginning, the direction of the handkerchief shaking is the same as the direction of the turning of the prayers of all sects. When men and women dance together in Guotzhuang, men and women should keep a certain distance to show respect and piety to women, and convey the cultural customs of "women are inferior to men and women are supreme" in ancient Eastern female culture. Qian Fuquan, inheritor of Manai guozhuang intangible cultural heritage, said: "In a fold of Guozhuang, men and women should in turn at least three pieces, and it may be repeated all night." So the end of Guozhuang folding is not arranged in advance, but decided the end by every eye contact.

In the field investigation, the author recorded 24 Guozhuang songs. Besides, this paper analyzes the typical Guozhuang songs of different sizes, while the rest are simplified and listed in the following article. It conducts from the material, mode, tonality, tone area and other aspects below.

1) Material and structure: Before all Guozhuang starts, you must first dance the folding of "Dong ber Zong", which is similar to the opening line of "Big Guozhuang", meaning a lot of happy things; There are two versions, and this version is one of them. There is no difference between the two versions. The structure chart is as follows (Table 1).

Table 1. Dong ber rong musical structure.

\begin{tabular}{cccccc}
\hline Period & $\mathrm{A}$ & $\mathrm{A}_{1}$ & $\mathrm{~B}$ & $\mathrm{~B}_{1}$ & $\mathrm{~B}_{2}$ \\
\hline Phrase & $\mathrm{a}+\mathrm{b}+\mathrm{a}^{1}+\mathrm{b}^{1}$ & $\mathrm{a}^{2}+\mathrm{b}^{2}+\mathrm{a}^{3}+\mathrm{b}^{3}$ & $\mathrm{c}+\mathrm{c}^{1}+\mathrm{d}+\mathrm{d}^{1}$ & $\mathrm{c}^{2}+\mathrm{c}^{3}+\mathrm{d}^{2}+\mathrm{d}^{3}$ & $\mathrm{c}^{4}+\mathrm{c}^{5}$ \\
Phrase & $5+4+4+3$ & $3+3+4+3$ & $5+5+3+4$ & $6+5+3+3$ & $6+6$ \\
structure & $5+4+4+5$ & & & \\
\hline
\end{tabular}


a for 1 to 5 sections; $\mathrm{b}$ is 6 to 10 sections; $\mathrm{a}^{1}$ for 11 to 15 sections 1 ; $\mathrm{b}^{1}$ for 16 to 18 sections; $\mathrm{a}^{2}$ for 19 to 21 sections 2 ; $\mathrm{b}^{2}$ for 22 to 24 sections; $\mathrm{a}^{3}$ for 25 to $28 \mathrm{sec}$ tions 3 ; $\mathrm{b}^{3}$ is sections 29 to 31 ; c is 32 to 36 sections; $\mathrm{c}^{1}$ is 37 to 41 sections; $\mathrm{d}$ is 42 to 44 sections; $\mathrm{d}^{1}$ is 45 to 48 sections; $\mathrm{c}^{2}$ is 49 to 54 sections 2 ; $\mathrm{c}^{3}$ is 55 to $59 \mathrm{sec}$ tions $3 ; \mathrm{d}^{2}$ is 60 to 62 sections; $\mathrm{d}^{3}$ is 63 to 65 sections; $\mathrm{c}^{4}$ is 66 to 71 sections; $\mathrm{c}^{5}$ is 72 to 77 sections. From the perspective of the internal structure relationship of the phrase, the data of $5+4$ and $4+3$ show that the phrase is irregular, cause the melody that changes and repeats in each sentence is based on the fixed melodic framework, and the singer shows off his skills and improvises, so the structure is relatively free. From the material point of view, passages $A_{1}, A_{2}$ and $A_{3}$ are reduced and changed repetitions developed from the material of $\mathrm{A}$; Dong Berrong is not a single material song, it is composed of four different materials: a, b, c and $d$. You can also think of $b$ as a phrase developed from a split, and $d$ as a phrase developed from c split. See example 2 for the four materials a, b, c and $d$ (the lowercase letters in the box are the marks of the materials).

Transcription 2: dong ber rong transcription.

\section{Dong ber Rong}
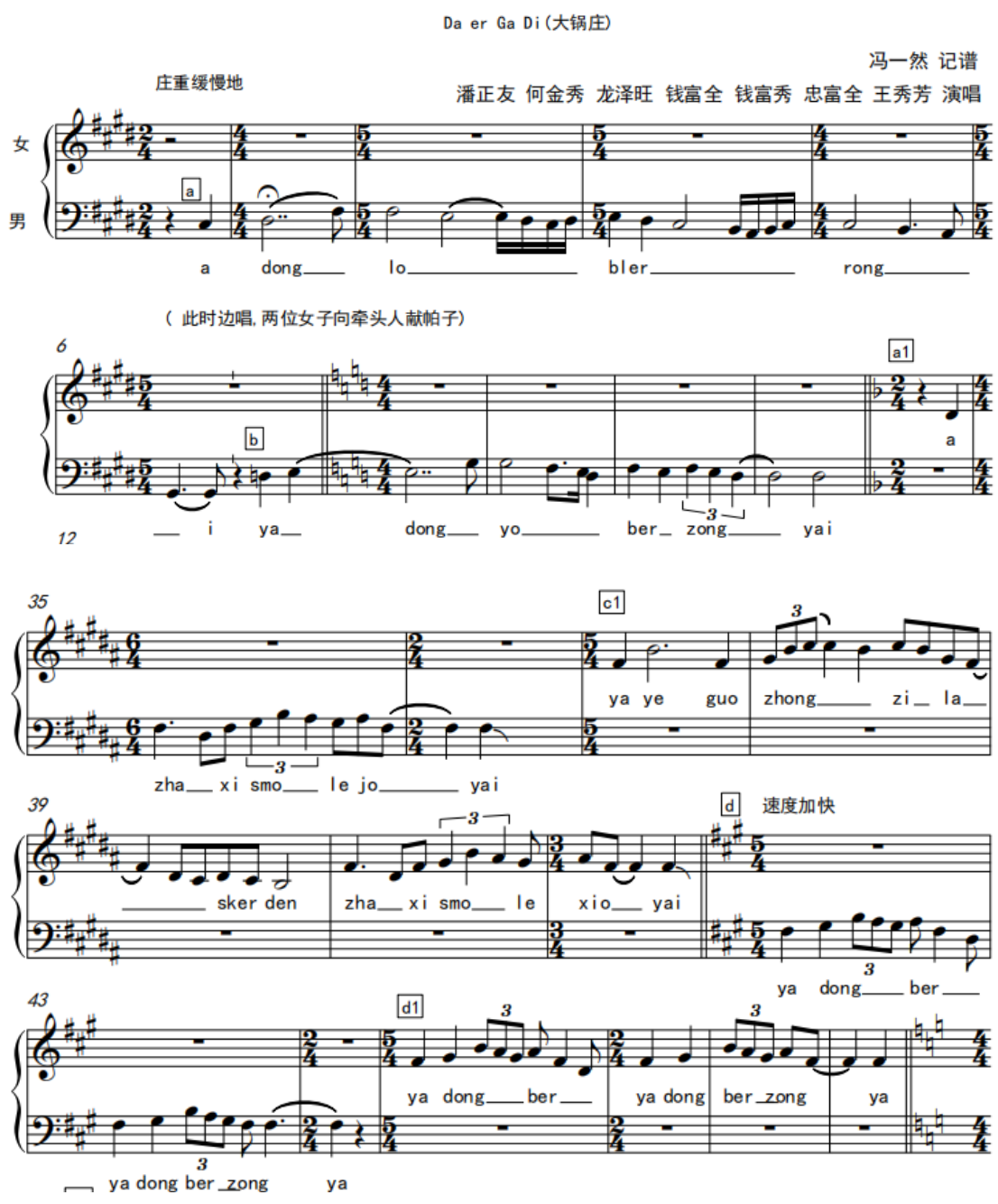
However, Jiamcuqiwer, a small Guozhuang dance performed during house reconstruction and happy events, is a segmental song developed from a single melodic material. The following score is the first paragraph, see example 3.

Transcription 3: jiam cu qi wer transcription

\section{Jiam Cu Qi Wer}
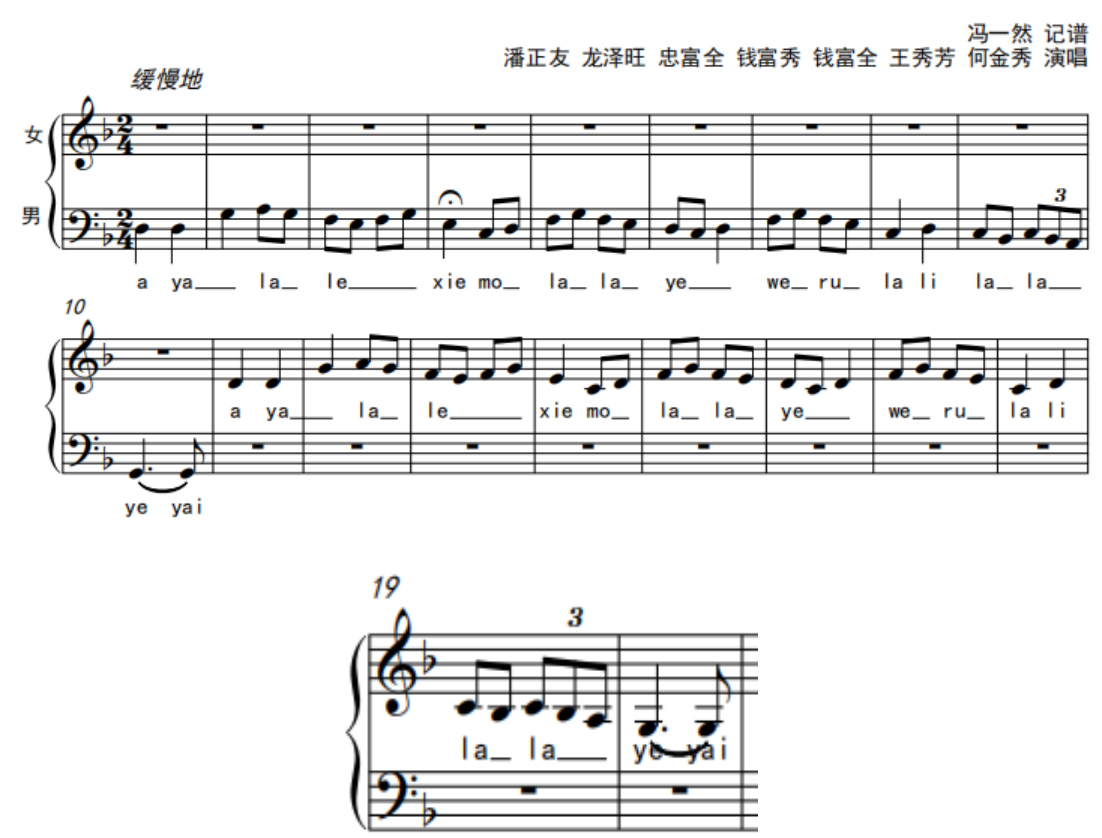

The whole song has a total of 24 paragraphs, all using the melody shown in the above example; the melody is simple and catchy, but the lyrics speak volumes. A happy life can be achieved as long as one hearts and one minds. In 24 verses, there is repetition, there is contrast, and there are structures similar to repetition. In order to demonstrate the rule of lyrics, the author named the first paragraph as $\mathrm{A}$, the second paragraph as the first paragraph, as a repetition of $\mathrm{A}$, the third paragraph is different from the first paragraph, named $B$, the sixth paragraph is $C$. In this way, the lyric structure of the first six paragraphs is: $A+A$ repetition $+\mathrm{B}+\mathrm{A}+\mathrm{B}+\mathrm{C}$. The first four paragraphs, according to the lyric material, are a trisection structure with reproduction, while the last three paragraphs are juxtaposed. The next eight paragraphs, each one different has an interesting connection: the lyrics are written in a similar way to the "Top truth", where the end of the last verse begins the next (Table 2).

Each lyric begins with o ya and ends with ye yai or yai. The author thinks Oya and yai are modal particles, similar to "hello" and "hey". It is o ya which means "yes" in today's Jiarong Tibetan. Paragraph 14 and paragraph 13 also have a connection with the "reality", and the lyrics of paragraph 14 are the same as that of the first paragraph A, and then after nine different lyrics of the "reality" relationship, it returns to the first paragraph and ends in the first paragraph. This lyric structure phenomenon is very close to a statement: "circle dance is a 
Table 2. Jiam cu qi wer lyrics structure.

\begin{tabular}{|c|c|}
\hline Phase 7 & Oya wa ne bo za shnemze ze, niamsguor sten guermashiedeYai. \\
\hline Phase 8 & $\begin{array}{l}\text { Oyaniamsguor stenguer ma shiede, niamla shken qierxiangShi } \\
\text { xiangyai. }\end{array}$ \\
\hline Phase 9 & Oyaniamlashgenqierxiangshi xiang,qi boqiongdi qionglayeyai. \\
\hline Phase 10 & Oya qi boqiongdi qiong la ye, mer jia sencuo de merz deyai. \\
\hline Phase 11 & Oyamer jia sencuodemerz de, yar qio senba den merz denyai. \\
\hline Phase 12 & Oya ya qio senba denmerz den, ni lema ba ser la shiyai. \\
\hline Phase 13 & Oyani lemabaser lashi, lali xiemolalayeyai. \\
\hline Phase $14=\mathrm{AO}$ & yalali xiemolalaye, werrulali lalayeyai. \\
\hline
\end{tabular}

manifestation of the Buddhist view of samsara." And this way of folding Guozhuang lyrics seems to reflect the theory of cause and effect in Buddhist teachings: The end of the former paragraph in lyrics is the beginning of the latter paragraph, the end of one life is the beginning of another life, and now the beginning of the mind determines the fate of the future. This is the embodiment on the ideology of samsara and causality in the form of music.

2) Tonality: Regarding the actual pitch notation, the tonality of Dong Ber Rong is relatively complex. Sentence a is composed of seven tones, so the tonality can be determined. As shown in the Table 3 below, sentence b consists of only four sounds $\mathrm{d}, \mathrm{e}, \mathrm{f}$ and $\mathrm{g}$, and there is no lift sign, so sentence $\mathrm{b}$ may be in the key of $\mathrm{C}$ or F. Since the inheritor may shift the melody by a minor second, it seems more reasonable that $\mathrm{b}$ should be in the key of $\mathrm{F}$. According to the key that determines A section: $a+b(E$ to $F) ; a^{1}+b^{1}\left(F \rightarrow{ }^{*} F\right) ; a^{2}+b^{2}\left({ }^{*} F \rightarrow G\right) ; a^{3}+b^{3}(G \rightarrow$ $\left.{ }^{\#} \mathrm{G}\right)$; Section B: $\mathrm{c}+\mathrm{c}^{1}$ (tone B); $\mathrm{d}+\mathrm{d}^{1}$ (A tone); Section B1 and B2 (tone C); There is a minor second increasing relationship in pitch between sentences a and $\mathrm{b}^{3}$, but the tonal change after paragraph $\mathrm{B}$ does not show the rule: $\mathrm{B} \rightarrow \mathrm{A} \rightarrow \mathrm{C}$ (tone). Jiamcu Qi Wer also raises the pitch by a minor second, and the other 24 songs also have the same phenomenon. The whole song shows the phenomenon of raising the pitch by a minor second piecemeal by sentence. So it's possible that the inherited higher mood of the people singing causes the melody to go up unconsciously. Jiamcu Qiwer is a song with a single tone if you ignore the minor second step up in the second creation.

The seven tones of the melody can be seen from the scale of sentence a (see example 2 for sentence a). If the seven notes appearing in the melody are arranged from the lowest to the highest, the seventh note $\mathrm{Si}\left({ }^{\#} \mathrm{~d}\right)$ will appear in the downbeat of the first bar, and the duration will take up three-quarters of the whole bar,the fourth tone fa (a), as a passing note with shorter duration, will appear once in the fifth beat of the third bar and once in the last beat of the fourth bar. The traditional seven tone si "variable palace" as a partial note, almost never appear in the downbeat position. Therefore, the mode of folding Guozhuang is different from the seven-tone national mode which takes fa and $x i$ as partial 
Table 3. Jiam cu qi wer mode and tonality.

\begin{tabular}{cccc}
\hline phrase & scale & initial phoneme & ending \\
\hline a & ${ }^{*} \mathrm{c}-{ }^{*} \mathrm{~d}-\mathrm{e}-{ }^{*} \mathrm{f}-{ }^{*} \mathrm{G}-\mathrm{A}-\mathrm{B}$ & $\mathrm{La}\left({ }^{*} \mathrm{c}\right)$ & $\operatorname{Mi}\left({ }^{*} \mathrm{G}\right)$ \\
$\mathrm{b}$ & $\mathrm{d}-\mathrm{e}-\mathrm{f}-\mathrm{g}$ & $\operatorname{Re}(\mathrm{d})$ & $\operatorname{Re}(\mathrm{d})$ \\
\hline
\end{tabular}

notes. Similarly, in Jiamcu Qiwer of small Guozhuang, there is also a long note $\mathrm{Si}$ (e) which is freely extended on downbeat, as shown in example 4, the first beat of the third bar. In other words, these two songs are composed of seven notes, and the Si note is not "partial". Compared with the "mild" seven-tone national mode, Both Dong Ber Rong and Jiamcu Qi Wer have a certain tension and mystery in the sound effect.

3) Range: The male vocal range of Dong Berrong consists of ${ }^{\#} G$ in the lowest tone and $\mathrm{d}^{1}$ in the highest tone, which is separated by an octave and a diminished fifth; The vocal range of the female voice consists of a sound in the lowest tone $g$ and $\mathrm{d}^{2}$ in the highest tone, which constitute a perfect fourth separated by an octave. The vocal range of the male voice in Jiamcu Qiwer consists of $G$ sound in the lowest tone big character group and $\mathrm{d}^{1}$ in the highest tone small character group, which constitute a perfect fifth separated by an octave. The vocal range of the female voice consists of $g$ sound and $\mathrm{d}^{2}$ sound, which are the lowest tone and the highest tone respectively, forming a perfect fifth separated by an octave. The pitch fluctuates greatly, and the double fold songs have the same range, giving people a sense of openness on the hearing.

Not only is Dong Berrong full of contrasts in tonality, material, the alternating male and female parts, but it is also elaborately arranged. Jiam Cu Qiwer is relatively common in the arrangement of voice parts, that is, the male voice takes part in one sentence and the female voice sings along to the last sentence at the end. There are also many interesting and worthy of analysis of Manai guozhuang songs. Due to the length of the song, it will not analyze all of them respectively. The following is the collected information and lyrics of 23 Manai Guozhuang songs.

Big Guozhuang:

1) Dong Ber Rong, happy events.

2) Namke chiaJi, nothing can grow without enough rain; The whole society will be stable if treating others in standard; Chieftain is a bridge to build friendship if uniting Yin Shan and Yang Shan (singing while shaking the shoulders like a bridge): the society cannot be healthy if the world without a wise ruler.

3) Ni Mo Na Chi, pregnant mother lives for nine months to give you birth, feed you breast milk and raise you grow up, now marrying her daughter, hoping the daughter could be grateful to her mother. Metaphor: The daughter should remember the kindness of raising and work hard in her husband's family. This is the Guozhuang led by the uncle. Since the daughter got married, the family will dance and cry.

4) Terjiqinbu, known as the second Guanyin Temple in Lhasa, it is tall and 
magnificent as a paradise for people to make pilgrimages to pray for well-being.

5) Song jian rong bu, the children and grandchildren are thriving like towering trees with luxuriant branches and deep roots. Metaphor: On the happy day, the elders give golden words to the younger generation and sing the blessings of Guozhuang.

6) Wan Zen Ren Sa, relatives take care of each other for a long time, help each other in difficult times, know how to be grateful to predecessors, and remember each other from generation to generation.

7) "Nial Ce Pangdang", the house has support, singing about the house is strong and warm. When the high officials and nobles are building house, big masons will lead jump a fold Guozhuang.

Small Guozhuang:

8) Jiamcu qi we, although the sparrow is small and fully equipped, as long as we work together, a happy life can be realized.

9) "Lasa Gongbi", send off the Lama to Nyingchi Tibet for further study.

10) "Ne Jiampa Wang", praise the life of equality: roc bird and pheasant could both lay eggs, long chopsticks and short chopsticks are used for the same purpose.

11) "Qie Mo Qiang Dong", when building a house, mixed wine is heated in the middle of the circle dance, one pot for each person, starting with the leader in turn.

12) "Shegu Dar de" is a song sung by two couples who get married at the husband's house, singing about the prosperity of the family, the unity of the relatives, and the luxuriation of the deep roots. According to the Jiarong Tibetan Folk Custom, "Skura Ruda" is the legendary name bestowed by the merdor mountain god to Siguniang Mountain, which means the god of mountain protection. (Mao, 2011) 59 the song is named after the mountain, which is also the embodiment of nature worship in the primitive beliefs of Bon.

13) Yaol Lil Qing imesno, my place and my girl (the last song to be sung when sending her away) advise her not to marry far away.

14) ge la dong jian, we are blowing the noble and rare conch, men and women dressed in silk and satin, living a beautiful life.

15) "Lasa Jie Wu" tells the process of bringing back a lion from Tibet, it is a tribute to panchen's small Guozhuang.

16) Ni Shi Gemqier, it celebrates the fruit and petals of the wild cherry.

17) Kenmjierwa bu, the branches of the water wax gourd are the most developed like a handsome boy. How to get milk step by step.

18) $\mathrm{Ma}$ da ser jia wu, on a wide and flower-filled lawn, everyone danced a lively Guozhuang dance.

19) "Ga Ra wa Bu", the whole process of buying cattle from the farming area to the pastoral area, a total of more than 30 paragraphs, each paragraph of the words are different (narrative) metaphor: the life away from home is very hard.

20) Na Ke ne La (A song of Guozhuang) cannot stop thinking of you, not afraid of all obstacles, watching you disappear sadly. 
21) Wen be na dong, Where did the felt hat (a hat made of wool that is typical of men's clothing) come from? Where did the veil come from?

22) "Ba Wong Ni Za" eulogizes the Tibetan young man of Jiarong.

23) "Ci La Songsong” eulogizes the moon love theme small Guozhuang.

\section{Endnotes}

It can observe the cultural foundation in the music structure through Manet Guozhuang while music is a form of culture and ideology, which embodies the teachings of Buddhism and Bon religion everywhere. The ideology of Buddhism's view of rebirth is not only reflected in the dance form of circle dance, but also in the musical structure of Manai Guozhuang. The true creation technique in the lyrics of Jiamcu Qiwer seems to be in line with the theory of cause and effect in Buddhist teachings: the end of the previous lyrics is the beginning of the next lyrics, the end of one life is the beginning of another, and the inspiration of the present decides the fate in the future.

The trichotomy of music structure $(\mathrm{A}+\mathrm{B}+\mathrm{A}, \mathrm{A}+\mathrm{B}+\mathrm{C})$ is the embodiment of the concept on "three realms" of Bon. The material of Manai Guozhuang's songs is closely related to nature and life. Some praise cherry fruits and the moon, and others like Shegu Darde, which is named after a mountain, praise the deep roots of their relatives. From the theme of the songs, it can find out the ancestors' belief and worship of nature under the ideology of the Bon Religion.

There are also many aspects of contrast and unity in the music structure: tonals, the contrast between male and female parts and materials, as well as the echo in the structure. In the music creation, the ingenious idea of the Bon buddhist ritual is integrated everywhere. People have to admit that the wisdom of the Tibetan ancestors of Jiarong is no less than that of the European musicians of the same time.

Above all, the rich connotation of Manai Guozhuang is where its charm lies. The art form that has charm, connotation and can withstand scrutiny can be remembered and inherited by people for a longer time. Therefore, it is hoped that the research results of this paper can make a contribution to the inheritance and protection of Manai Guozhuang intangible cultural heritage.

Finally, I would like to thank the inheritors Qian Fuquan, Zhong Fuquan, Qian Fuxiu, Pan Zhengyou, He Jinxiu and Long Zewang for singing more than 20 Manai Guozhuang songs in the hot summer, especially grandpa Zhong Fuquan who lives on the top of the mountain. Every interview, he had to walk several miles from the top of the mountain to the village government at the foot of the mountain. At the same time, I would like to thank my tutor Professor Tan Yong, deputy dean of school of Art, Southwest University for Nationalities, for his diligent guidance, as well as corresponding author Jin Shaona.

\section{Supported}

This article is supported by the Fundamental Research Funds for the Central 
Universities, Southwest Minzu University in 2019, Project No. 2019 SQN28.

\section{Conflicts of Interest}

The author declares no conflicts of interest regarding the publication of this paper.

\section{References}

Ga, J. (2005). Study on the Structure and Morphology of Tibetan Traditional Music. Shanghai Conservatory of Music.

Mao, L., \& Li, Z. J. (2011). Jiarong Tibetan Folk Customs. Minzu University of China Press.

Yang, X. F. (2009). Field Investigation of "Manai Guozhuang". Chinese Music, No. 3, 25-30. 